\title{
THEM: Ferramenta colaborativa para suporte a avaliações de interfaces baseadas na Avaliação Heurística
}

\author{
Francis Santos ${ }^{1}$, Glívia A. R. Barbosa ${ }^{1}$, Ismael S. Silva ${ }^{1}$, Flávio R. S. Coutinho ${ }^{1}$ \\ ${ }^{1}$ Departamento de Computação - Centro Federal de Educação Tecnológica de Minas \\ Gerais (DECOM/CEFET-MG). Av. Amazonas, 7675 - Nova Gameleira - Belo \\ Horizonte, MG - Brasil. \\ francissantos8@gmail.com, gliviabarbosaldecom.cefetmg.br, \\ ismaelsantana@decom.cefet.br, coutinho@decom.cefetmg.br
}

\begin{abstract}
This paper presents THEM, a collaborative solution that seeks to assist in the evaluation process of interfaces based on Heuristic Evaluation. Experiments have shown that THEM is a useful and satisfactory tool for its purpose. Proposing tools such as THEM is relevant because, in addition to automating steps and artifacts during the evaluation of heuristic-based interfaces, it encourages the development and evaluation of other tools that seek to support not only interface evaluation methods, but other processes related to software development.
\end{abstract}

Resumo. Esse trabalho apresenta a THEM, uma solução colaborativa que busca auxiliar no processo de avaliação de interfaces baseado na Avaliação Heurística. Os experimentos realizados demonstraram que a THEM é uma ferramenta útil e satisfatória mediante o seu objetivo. Apresentar ferramentas como a THEM é relevante porque, além de automatizar etapas e artefatos durante a avaliação de interfaces baseada em heurísticas, incentiva o desenvolvimento e a avaliação de outras ferramentas que buscam suportar não apenas os métodos de avaliação de interface, mas outros processos relacionados ao desenvolvimento de software.

\section{Introdução}

O crescente desenvolvimento tecnológico e a demanda por soluções de Tecnologia da Informação (TI) que potencializam a experiência satisfatória dos usuários (do inglês, User Experience - UX), têm exigido da Engenharia de Software constantes melhorias em seus processos, métodos e suas técnicas [Chaves, 2010]. Nesse sentido, cada vez mais, as atividades de desenvolvimento de software exigem pessoas treinadas, processos bem definidos e ferramentas adequadas [Chaves, 2010].

Contudo, segundo Chaves (2010) e Pressman (2011), ainda existe uma carência de ferramentas, bem difundidas, em algumas áreas relacionadas à produção de software. Essa carência faz com que algumas etapas do desenvolvimento de software sejam realizadas de forma manual ou com ferramentas inadequadas (e.g., ferramentas não específicas), tornando os processos de desenvolvimento, gestão e qualidade de software menos produtivos e mais propensos a erros [Chaves, 2010][Pressman, 2011].

Uma das etapas do desenvolvimento de software que possuem pouco suporte de ferramentas é a avaliação de interfaces por inspeção. Nessa etapa, o modelo de interface e interação do software desenvolvido é inspecionado e avaliado, sob a perspectiva de 
especialistas, para identificar potenciais problemas que os usuários podem experimentar durante a interação com o sistema [Prates e Barbosa, 2003].

Na literatura é possível encontrar métodos de inspeção de interface que focam na avaliação de diferentes qualidades de uso, como a usabilidade (e.g., Avaliação Heurística, Percurso Cognitivo), a acessibilidade (e.g., avaliadores automáticos de acessibilidade - daSilva e Hera ) e a comunicabilidade (e.g., Método de Inspeção Semiótica (MIS)) [Prates e Barbosa, 2003][Barbosa e Silva, 2010].

Embora esses métodos não envolvam a participação dos usuários durante sua execução, a aplicação dos mesmos é de extrema importância durante o desenvolvimento de software, uma vez que permitem antecipar e analisar os problemas no projeto de interface que podem afetar diretamente a usabilidade, acessibilidade, comunicabilidade e outras qualidades de uso que impactam na experiência satisfatória do usuário [Prates e Barbosa, 2003].

Um dos métodos de avaliação de interfaces por inspeção mais difundido é a Avaliação Heurística (AH) [Nielsen, 1994]. Por meio desse método, um grupo de avaliadores colabora e julga o modelo de interface e interação de um software a partir de um conjunto de 10 heurísticas de usabilidade. Além disso, segundo seu proponente, o método prevê a possibilidade de incluir novas heurísticas de avaliação ou adaptar as existentes, sejam elas para avaliar a usabilidade ou outras qualidades de uso (e.g., acessibilidade) [Nielsen, 1994].

Apesar de exigir conhecimento dos avaliadores para sua aplicação, o método Avaliação Heurística é considerado didático e de simples execução, o que o torna adequado tanto para ambientes acadêmicos, quanto para ambientes corporativos [Nielsen, 1994][Prates e Barbosa, 2003][Barbosa e Silva, 2010].

Existe uma demanda de ferramentas que auxiliem no processo de execução e análise da Avaliação Heurística [Prates e Barbosa, 2003][Chaves, 2010][Pressman, 2011]. Isso porque, embora seja possível encontrar ferramentas que visam auxiliar à execução de avaliações por inspeção (e.g., APIU [Santos, 2011], ISPIS [Kalinowski et al. 2004], IBIS [Lanubile et al., 2003], MISTool [Jesus e Silva, 2010], Heva [Oeiras et al., 2008]), a maioria delas não apoia ou limita o suporte ao método [Oeiras et al., 2008]. Por exemplo, a ferramenta Heva limita sua execução por meio do navegador Firefox. Isso é um problema porque restringe a execução da Avaliação Heurística apenas para sistemas web [Oeiras et al., 2008].

Considerando a aplicabilidade da Avaliação Heurística, tanto no âmbito acadêmico, quanto no coorporativo para avaliar o modelo de interface e interação de sistemas e a demanda por ferramentas que suportem a execução colaborativa desse método, o presente trabalho tem como objetivo apresentar a Tool for Heuristic Evaluation Methods (THEM), uma ferramenta colaborativa que auxilia nas avaliações de interfaces baseadas na Avaliação Heurística (AH).

A ferramenta foi desenvolvida de modo que suas funcionalidades contemplassem as principais etapas e artefatos da Avaliação Heurística e que os avaliadores pudessem colaborar durante a avaliação. Finalizado o desenvolvimento, a THEM foi avaliada e os resultados permitiram demonstrar sua utilidade e adequação ao uso na perspectiva de seus usuários. 
Desse modo, esse trabalho é relevante porque, além de suportar todas as etapas da Avaliação Heurística e contribuir com a automação de atividades previstas nesse tipo de avaliação (e.g., geração de gráficos e relatórios), a THEM motiva novos trabalhos relacionados que visam desenvolver e delinear as vantagens e desvantagens de ferramentas que suportam a execução dos métodos de avaliação de interface.

\section{Trabalhos Relacionados}

$\mathrm{Na}$ literatura foi possível encontrar trabalhos que apresentam ferramentas para suportar processos de avaliação por inspeção em diferentes perspectivas. Por exemplo, as ferramentas GrIP, IBIS e ISPIS, propostas respectivamente por Halling e outros (2002), Lanubile e outros (2003) e Kalinowski (2004) foram desenvolvidas para apoiar a inspeção de artefatos de software produzidos ao longo de todo o processo de desenvolvimento (e.g., documentação, código fonte, interfaces). Em outras palavras, as ferramentas não foram construídas especificamente para avaliação de interfaces de usuários por inspeção.

No âmbito de softwares para suportar as avaliações de interfaces por inspeção é possível encontrar ferramentas como a TOWABE [Itakura e Vergilio, 2002], SUIT [Ardito et al., 2006], Heva [Oeiras et al., 2008], APIU [Santos, 2011], MISTool [Jesus e Silva, 2010] e ApplicSIM [Dias e Prates, 2013]. Contudo, a maioria delas não foca nas avaliações por inspeção guiadas por heurísticas, como a Avaliação Heurística.

Por exemplo, as ferramentas TOWABE e SUIT suportam o processo de inspeção de usabilidade baseado na técnica Systematic Usability Evaluation (SUE). Essa técnica guia as atividades dos inspetores por meio de tarefas que descrevem, com precisão, os objetos da aplicação a serem procurados e quais ações devem ser executadas durante a inspeção para analise de tais objetos [Itakura e Vergilio, 2002][Ardito et al., 2006].

Por sua vez, a Ferramenta APIU permite executar a inspeção de usabilidade, independente do método de avaliação. Contudo, os experimentos com a ferramenta indicaram que o principal diferencial da APIU se mostrou um empecilho para sua utilização, já que sua generalidade em relação aos métodos de inspeção a tornaram pouco usual [Santos, 2011]. Nesse sentido, Santos (2011) argumenta sobre a importância de oferecer ferramentas flexíveis, porém que estejam alinhadas com métodos de avaliação por inspeção específicos.

Nessa linha, as ferramentas MISTool [Jesus e Silva, 2010] e ApplicSIM [Dias e Prates, 2013] foram propostas para apoiar a execução do Método de Inspeção Semiótica (MIS). O MIS é um método de inspeção que avalia a comunicabilidade da interface, que não faz uso de heurísticas durante a avaliação [de Souza et al., 2006].

Por sua vez, a ferramenta Heva, apresentada por Oeiras e outros (2008), provê suporte à aplicação da Avaliação Heurística. Segundo Oeiras e outros (2008), a ferramenta foi criada para minimizar a sobrecarga de trabalho que o processo manual de execução do método exige (e.g., obtenção dos dados e consolidação de relatórios). Os experimentos iniciais com a ferramenta apontaram erros e problemas de usabilidade que demandaram correções. Além disso, os autores ressaltaram que a Heva demanda por evoluções, uma vez que está limitada por ser uma extensão do Mozilla Firefox, permitindo apenas a avaliação de aplicações web compatíveis com esse navegador [Oeiras et al., 2008]. 
A partir dos trabalhos relacionados foi possível constatar que, embora as ferramentas APIU e Heva possam ser utilizadas durante avaliações de interfaces baseadas na Avaliação Heurística, apenas a Heva foi proposta especificamente com essa finalidade. Além disso, a Heva limita sua utilização apenas para a avaliação de sistemas web, compatíveis com o navegador Firefox. Diante desse cenário, o presente trabalho se difere dos demais porque apresenta a THEM, uma ferramenta colaborativa para apoio à aplicação e análise de avaliações de interfaces por inspeção baseadas na Avaliação Heurística. A THEM independe da plataforma do sistema a ser avaliado e auxilia em todo o processo de avaliação, desde a coleta de dados até a geração de relatórios consolidados.

\section{Metodologia e Referencial Teórico}

Conforme ressaltado por Chaves (2010), para que uma ferramenta auxilie adequadamente uma determinada fase do desenvolvimento de software, a solução proposta precisa ser aderente às atividades e artefatos que essa fase demandam [Chaves, 2010]. Por isso, a metodologia proposta para condução desse trabalho consistiu, inicialmente, em definir os requisitos e a arquitetura da ferramenta THEM.

Os requisitos foram identificados a partir da especificação do método Avaliação Heurística, que fundamenta o fluxo de execução da ferramenta, e foram validados por 05 especialistas com mais de 10 anos de experiência na execução desse método. Na sequencia, a THEM foi desenvolvida a partir dos requisitos levantados e da arquitetura definida. Posteriormente a ferramenta foi avaliada junto aos seus usuários para verificar sua adequação ao uso por meio de Testes de Usabilidade [Rubin e Chisnell, 2008].

A seguir será apresentado o método Avaliação Heurística, que fundamenta as funcionalidades, bem como o fluxo de execução da ferramenta THEM.

\subsection{Avaliação Heurística}

Avaliação Heurística é um método de avaliação de interface por inspeção baseada na opinião de especialistas apresentado por Nielsen (1994). Esta avaliação tem como base um conjunto de heurísticas definidas por Nielsen (1994), que estabelecem as características desejáveis para interação com o software e as melhores formas de organização de sua interface para potencializar a usabilidade.

As heurísticas definidas por Nielsen (1994) são: (1) Visibilidade do estado do sistema; (2) Correspondência entre o sistema e o mundo real; (3) Controle e liberdade do usuário; (4) Consistência e padronização; (5) Reconhecimento ao invés de memorização; (6) Flexibilidade e eficiência no uso; (7) Design estético e minimalista; (8) Ajudar usuários no reconhecimento, diagnóstico e na recuperação de erros; (9) Prevenção de erros e (10) Ajuda e documentação.

Entretanto, Nielsen (1994) alerta que, diante da evolução tecnológica, as heurísticas utilizadas durante a avaliação podem ser adaptadas e/ou incrementadas conforme o julgamento dos especialistas e a necessidade do domínio da avaliação [Nielsen, 1994][Prates e Barbosa, 2003]. Normalmente, essas heurísticas são adaptadas conforme os domínios dos softwares analisados (e.g., interfaces de sistemas web demandam por heurísticas complementares aos sistemas desktop) [Prates e Barbosa, 2003]. 
O método deve ser executado em 3 etapas de forma colaborativa, Nielsen (1994) recomenda que o processo seja conduzido por três a cinco avaliadores, [Nielsen, 1994][Prates e Barbosa, 2003]. Inicialmente ocorre a etapa de preparação, na qual os avaliadores se familiarizam com o domínio do sistema a ser avaliado, bem como com os usuários que eles devem representar. Nesta etapa, também são organizadas as telas do sistema ou os seus protótipos, bem como as heurísticas a serem utilizadas na avaliação, conforme o escopo delimitado pelos avaliadores [Nielsen, 1994][Prates e Barbosa, 2003].

Em seguida, os avaliadores inspecionam as interfaces em sessões curtas (1 a 2 horas) julgando sua conformidade com as heurísticas de avaliação, procurando por potenciais problemas. Durante a inspeção, para cada problema, os especialistas devem registrar: (1) o local onde foi encontrado, (2) a(s) heurística(s) violada(s) - sendo que o mesmo problema pode violar mais de uma heurística, (3) a justificativa do problema e (4) sua gravidade [Nielsen, 1994][Prates e Barbosa, 2003].

A gravidade (ou severidade) dos problemas encontrados auxiliam no estabelecimento de um custo/benefício da sua correção e na priorização dos esforços para seu reparo ou reprojeto. Segundo Nielsen (1994), esse julgamento envolve os fatores de frequência da ocorrência do problema, o impacto do problema para a experiência do usuário e a persistência do problema no decorrer da interação do usuário.

Nielsen (1994) sugere uma escala de gravidade de 1 a 5, na qual: G1 - Não é um problema; G2 - Problema cosmético; G3 - Problema pequeno; G4 - Problema grande e G5 - Problema catastrófico. Contudo, assim como as heurísticas de avaliação, os níveis de gravidade também podem ser adaptados conforme o escopo da avaliação.

Finalmente, os avaliadores discutem e analisam os problemas identificados de forma consolidada. Ao fim dessa etapa, é gerado um relatório conclusivo, unificado, com todos os problemas encontrados, bem como o parecer dos avaliadores sobre a qualidade de uso (e.g., usabilidade) do sistema [Nielsen, 1994][Prates e Barbosa, 2003].

\section{Proposta de uma Ferramenta para Suporte a Avaliações de Interfaces baseadas na Avaliação Heurística}

Esta seção apresenta os requisitos que guiaram o desenvolvimento da THEM, bem como sua arquitetura e a ferramenta criada.

\subsection{Requisitos e Arquitetura da THEM}

Conforme informado anteriormente, a ferramenta aqui proposta tem como objetivo principal suportar as fases e os artefatos gerados durante avaliações de interfaces baseadas no método Avaliação Heurística. Diante desse objetivo, as principais funcionalidades e a arquitetura da THEM foram definidas a partir da descrição da Avaliação Heurística e validadas por 05 especialistas com mais de 10 anos de experiência na execução desse método. Desses especialistas, três executam o método em ambientes corporativos e dois o executam no ambiente acadêmico (i.e., pesquisa e ensino).

A partir desse levantamento foi definido que a THEM deveria contemplar as seguintes funcionalidades: (1) Gerenciar Usuários; (2) Autenticar no Sistema; (3) Gerenciar Projeto; (4) Adicionar Avaliador; (5) Definir Heurísticas da Avaliação; (6) 
Estabelecer Níveis de Gravidade; (7) Cadastrar Problema Encontrado; (8) Editar Problema; (9) Visualizar Resumo da Avaliação; (10) Gerar Relatório Individual; (11) Consolidar Projeto e (12) Gerar Relatório Final.

Além disso, seguindo a definição do método, foi definido que o público alvo da ferramenta é representado por qualquer usuário da área de Computação, que deseja executar avaliações de interfaces utilizando métodos baseados na Avaliação Heurística [Nielsen, 1994][Prates e Barbosa, 2003].

Em relação à arquitetura, a ferramenta foi desenvolvida na plataforma Web, utilizando a linguagem de programação C\#, o framework ASP.NET e o sistema gerenciador de banco de dados MSSQL Server. Essas tecnologias foram adotadas porque são robustas e amplamente utilizadas [Gupta et al., 2012]. Além disso, a THEM segue o padrão arquitetural Model-View-Controller (MVC), que favorece a organização do código e a evolução da ferramenta [Gupta et al., 2012]. A seguir a THEM será apresentada.

\subsection{Tool for Heuristic Evaluation Methods (THEM)}

A ferramenta THEM contempla um fluxo de execução similar aos passos da Avaliação Heurística. Desse modo, os avaliadores, depois de autenticados na ferramenta, podem acessar os módulos que permitem: (1) criar/gerenciar um projeto de avaliação de interface, (2) gerenciar os avaliadores participantes do projeto, (3) definir as heurísticas e/ou os níveis de gravidade da avaliação, (4) reportar os problemas identificados durante a avaliação e (5) gerar relatórios da avaliação. Além disso, a THEM dispõe de um sistema de ajuda para auxiliar os usuários durante sua utilização.

É importante ressaltar que os módulos da THEM, relacionados a um determinado projeto de avaliação, podem ser acessados conforme a necessidade dos avaliadores, em qualquer ordem, apesar de haver um fluxo de execução sugerido inspirado nas etapas da Avaliação Heurística.

Conforme demonstrado na Figura 1, as primeiras funcionalidades acessadas ao interagir com a ferramenta são a criação e a autenticação de usuários. Uma vez autenticado, o usuário acessa a página de gerência de projetos, conforme ilustrado na Figura 2. Por meio dessa interface, é permitido ao usuário a visualização e o cadastro de projetos de avaliação de interface no sistema, além de gerenciar convites enviados para participação em projetos de avaliação de outros usuários da THEM. Caso o usuário cadastre o projeto de avaliação, ele recebe o status de "avaliador moderador" do projeto criado.

Ao acessar um projeto especifico, o usuário é direcionado para a interface, "Menu do Projeto", ilustrado na Figura 3. A partir desse menu é possível personalizar a avaliação de acordo com as necessidades do avaliador (i.e., definir avaliadores, heurísticas, gravidades, bem como registrar os dados da avaliação e gerar relatórios). Nesta interface é permitido o acesso a todas as funcionalidades do sistema em qualquer momento da avaliação. 

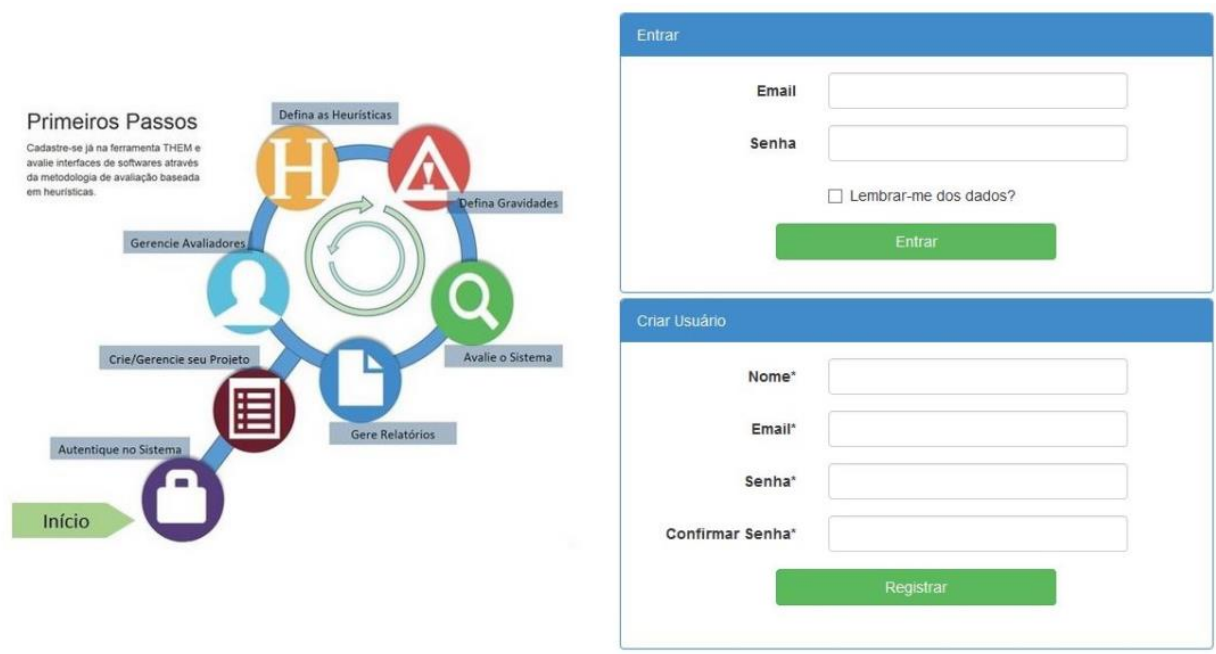

Figura 1. Página Inicial da THEM
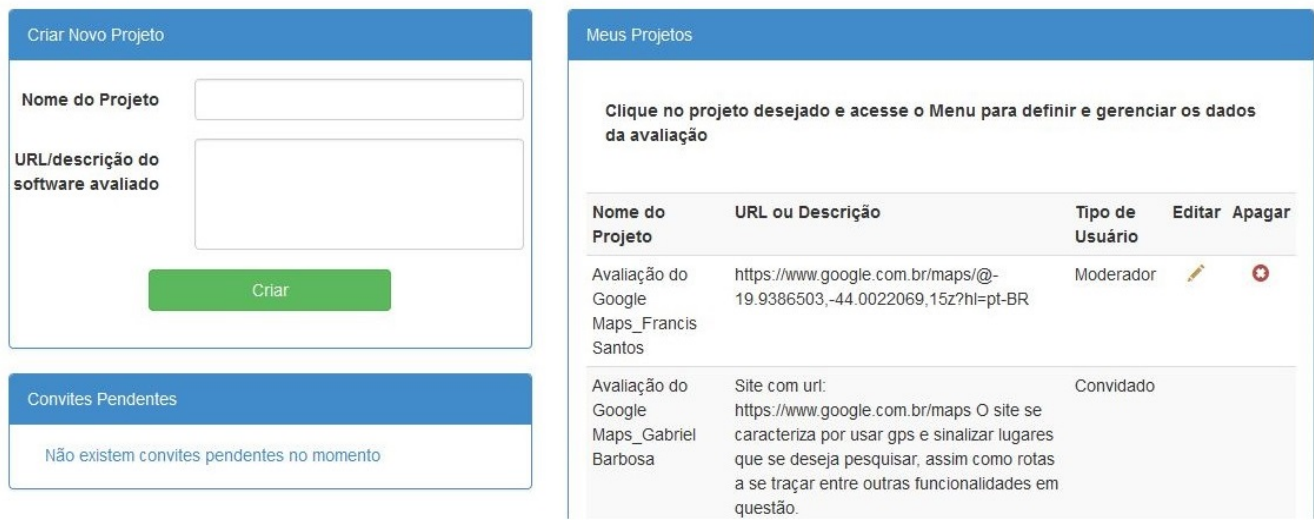

Figura 2. Gerência de Projetos de Avaliação de Interface

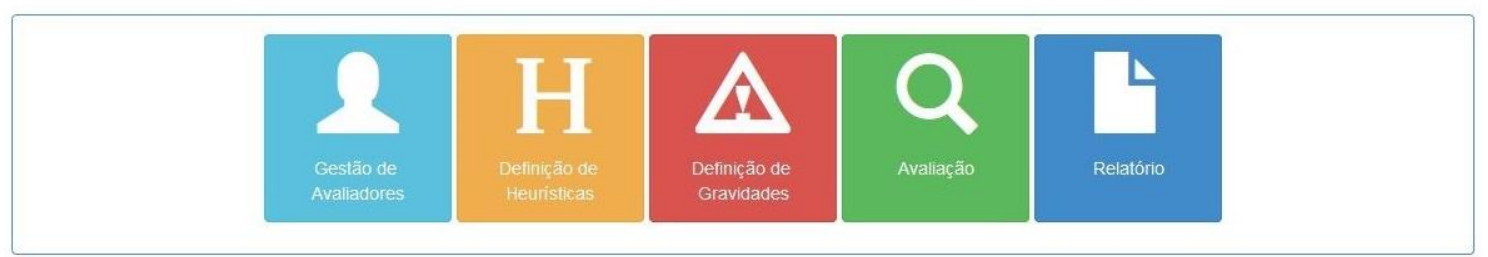

< Voltar para Minhas Avaliações

Figura 3. Menu de um Projeto de Avaliação de Interface

O modulo "Gestão de Avaliadores", demonstrado na Figura 4, permite ao avaliador moderador (i.e., avaliador que criou o projeto que está sendo gerenciado), buscar e convidar outros usuários da THEM para compor o seu grupo de avaliadores, bem como gerenciá-los. Todos os avaliadores adicionados ao projeto recebem, automaticamente, o status de avaliadores convidados. Para moderar a colaboração, um avaliador convidado não possui acesso aos módulos de "Definição de Heurísticas", "Definição de Gravidades", e "Gestão de Avaliadores", e utilizam de forma controlada o módulo "Avaliação", não sendo permitido, por exemplo, a remoção ou edição de problemas que não foram cadastrados por ele mesmo. 


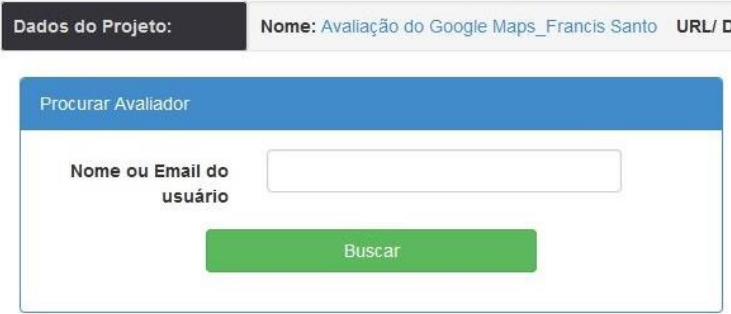

< Voltar para Projeto

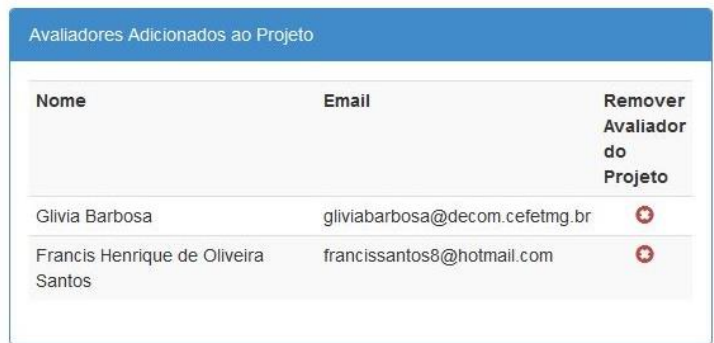

Figura 4. Gestão de Avaliadores

Definido o grupo de avaliadores do projeto de avaliação, o moderador tem a opção de definir as heurísticas que serão utilizadas durante a avaliação, conforme demonstrado na Figura 5. É importante ressaltar que, ao criar um novo projeto, as heurísticas e as gravidades de Nielsen (1994) são definidas como padrão na THEM para execução da avaliação.

Contudo, a THEM oferece diferentes opções para que as heurísticas utilizadas na avaliação sejam definidas, de modo que, se desejar, o moderador pode remover uma ou mais heurísticas de Nielsen (1994), adicionar novas heurísticas ao projeto, bem como reaproveitar heurísticas criadas em outros projetos de avaliação.

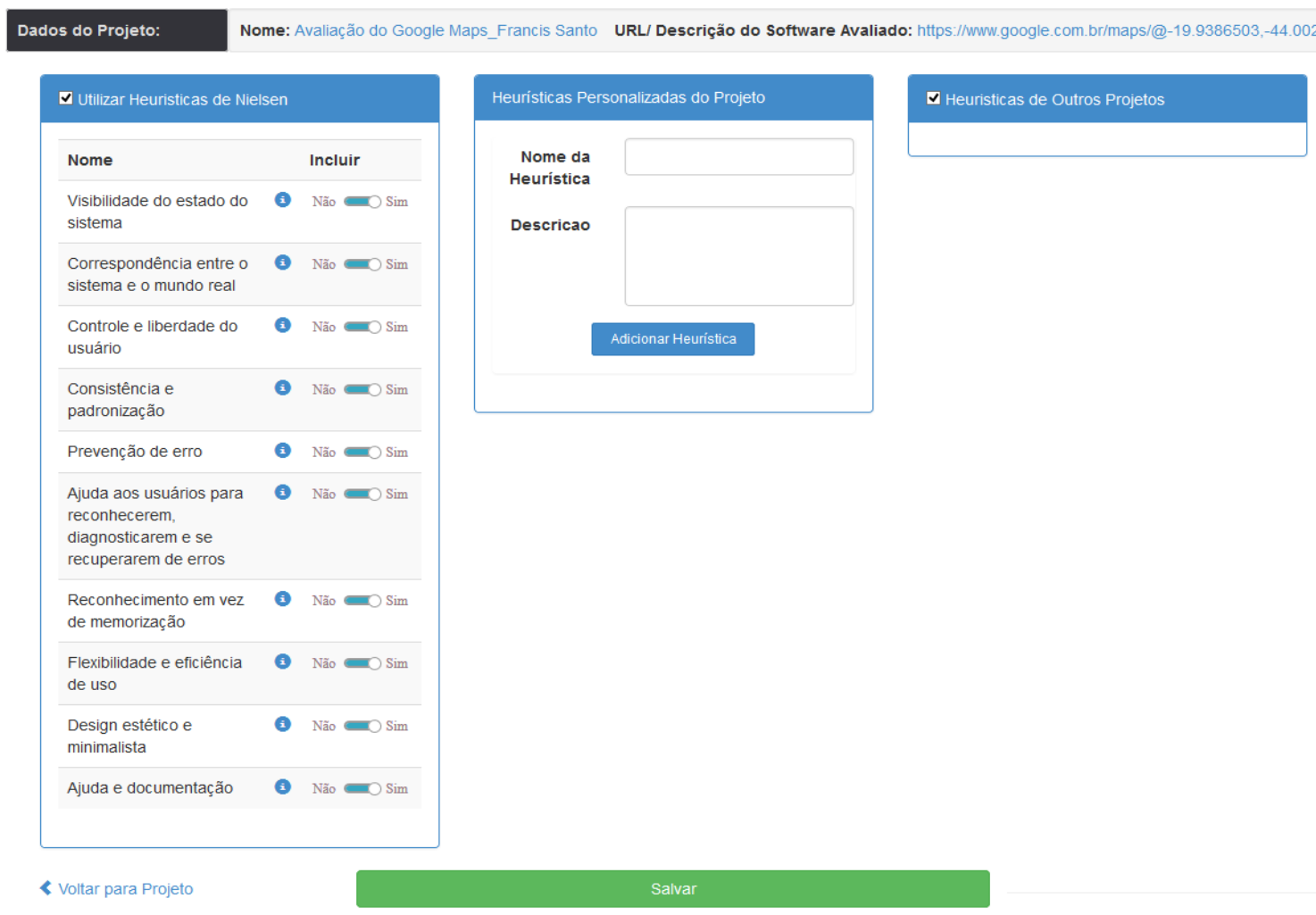

Figura 5. Definição das Heurísticas de Avaliação

Similar à definição de heurísticas, conforme ilustrado na Figura 6, o módulo "Definir Gravidades" permite a determinação das gravidades que serão associadas aos problemas encontrados durante a avaliação. Esse módulo permite a adição, remoção e 
edição das gravidades, e contém funcionalidades como o reestabelecimento das gravidades padrão e a ordenação das gravidades, conforme a necessidade do avaliador.

Dados do Projeto: Nome: Avaliação do Google Maps_Francis Santo URL/ Descrição do Software Avaliado: hittps://www.google.com.br/maps/@-19.9386503,-44.002

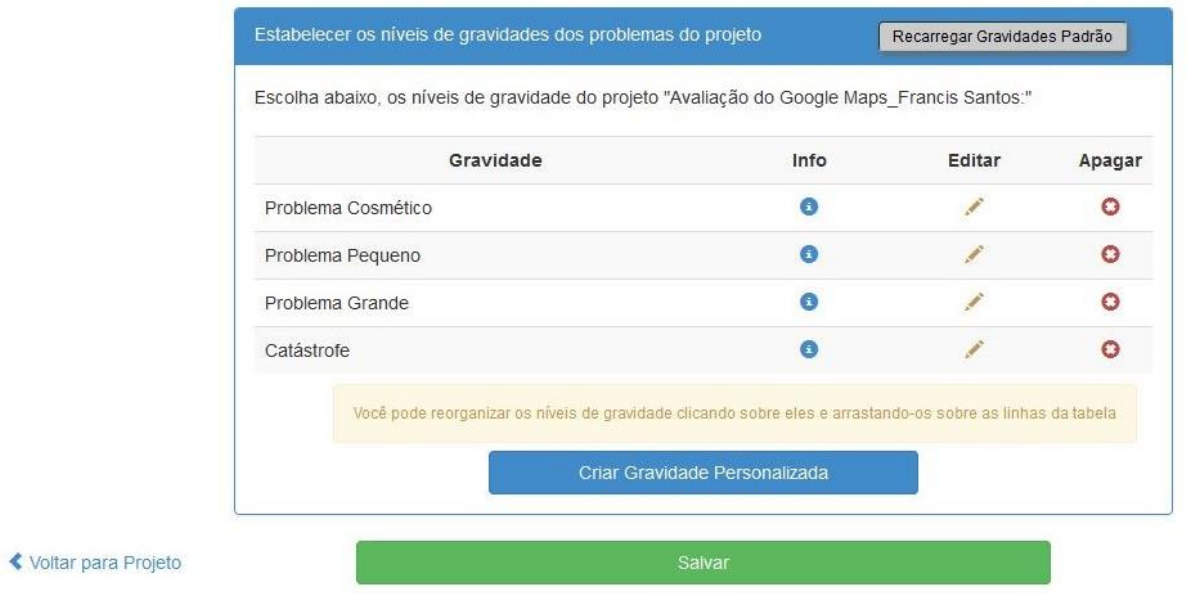

Figura 6. Definição das Gravidades da Avaliação

Finalizada a preparação para o projeto de avaliação de interface, os avaliadores podem executar a inspeção guiados pelas heurísticas e registrar os dados da análise utilizando a funcionalidade "Avaliação", disponível no "Menu do Projeto" na THEM. Conforme demonstrado na Figura 7, esse módulo contém as funcionalidades de cadastro e edição de problemas, visualização do resumo com indicadores da avaliação e consolidação do projeto.

Dados do Projeto: Nome: Avaliação do Google Maps_Francis Santo URL/ Descrição do Software Avaliado: https://www. google.com.br/maps/@-19.9386503,-44.0022C
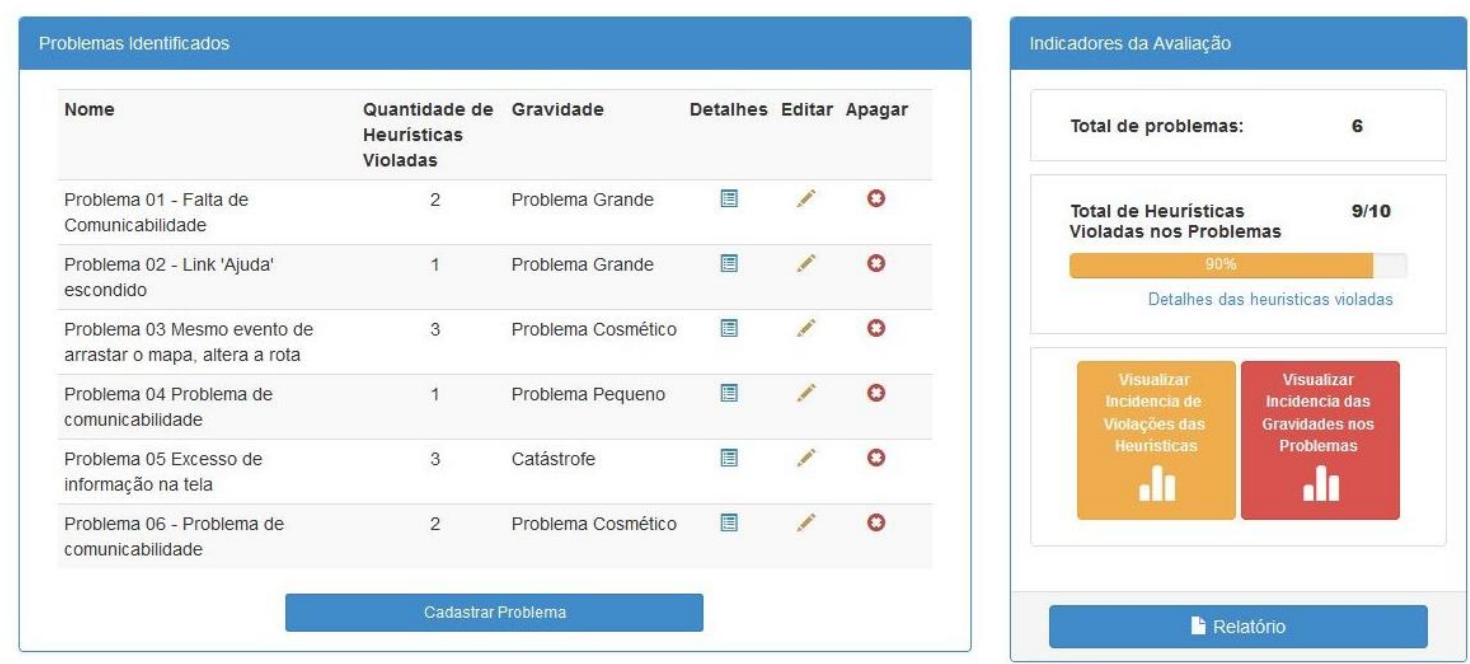

$<$ Voltar para Projeto

Figura 7. Módulo: "Avaliação de Interface”

O cadastro e a edição dos problemas são realizados por meio da interface ilustrada na Figura 8. A partir dessa interface os avaliadores informam o nome, a descrição e a gravidade do problema, as heurísticas violadas, bem como sua localização. A localização do problema é especificada por meio do upload de uma imagem que evidencia o local onde a violação se encontra. 


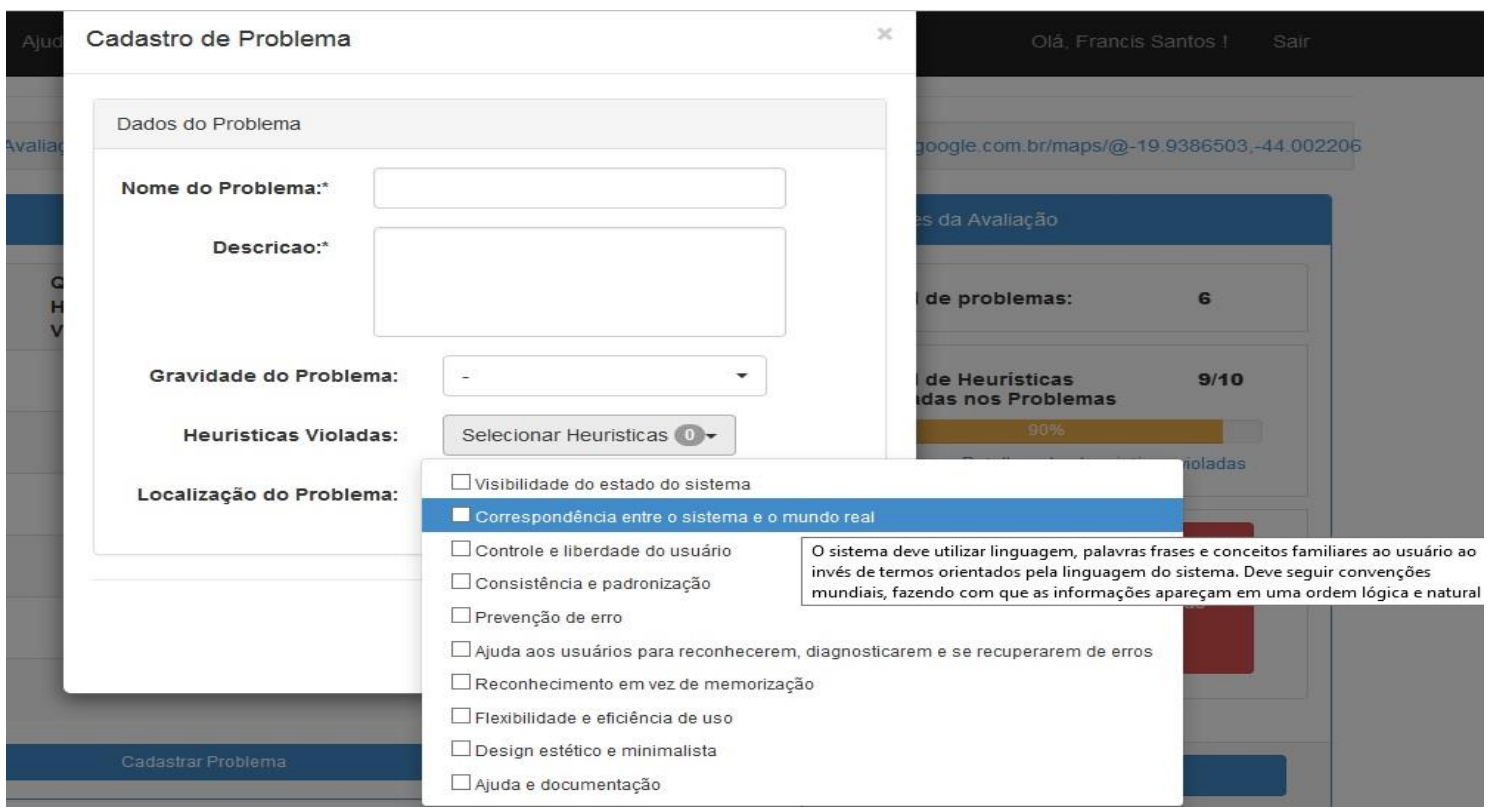

Figura 8. Cadastro de Problema na Interface

A visualização do resumo da avaliação é viabilizada por meio do bloco "Indicadores da Avaliação". Esse bloco possui dados e gráficos dinâmicos que indicam o total de problemas encontrados, o total de heurísticas violadas, a incidência da violação das heurísticas nos problemas e a incidência das gravidades nos problemas. Segundo os especialistas em avaliações de interfaces baseadas na Avaliação Heurísticas, esses dados são uteis para a análise e consolidação dos problemas [Nielsen, 1994][Prates e Barbosa, 2003].

A consolidação do projeto é feita pelo moderador, por meio da revisão dos problemas cadastrados pelos demais avaliadores. Para isso, o moderador deve observar os problemas no bloco "Problemas Identificados" e, removê-los ou alterá-los, observando questões como relevância e duplicidade. É importante salientar que, a ferramenta suporta a execução de avaliações de maneira colaborativa, de forma que todos os avaliadores visualizam os problemas cadastrados simultaneamente, ficando a cargo dos mesmos a verificação de duplicidades antes da inserção de problemas para reduzir o trabalho do moderador na consolidação do projeto.

A THEM possui ainda um módulo para personalizar e exportar o relatório da avaliação. Esse módulo pode ser acessado a qualquer momento pelo "Menu do Projeto" ou selecionando a opção "Relatório" na interface da "Avaliação" (Figura 7). Conforme demonstrado na Figura 9, além de apresentar os dados, bem como os problemas reportados durante a inspeção, o relatório final também pode ser complementado por meio de seções editáveis (e.g., Introdução; Resultados da Avaliação; e Conclusão). Além dessa possibilidade de personalização, utilizando a ferramenta, também é possível editar o relatório final depois de exportado, uma vez que ele é gerado em um formato de arquivo susceptível a tais alterações nos editores de textos (i.e., editores compatíveis com o formato docx). Um vídeo demonstrando as funcionalidades da THEM está

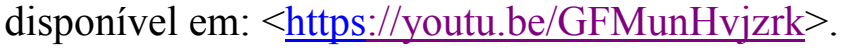




\section{Introduçäo}

Problemas Detectados

Resultados da Avaliação

Conclusão

< Voltar para Projeto

Figura 9. Relatório da Avaliação

Finalizado o desenvolvimento da THEM foi possível compará-la com as ferramentas similares encontradas na literatura. As ferramentas foram comparadas em relação aos parâmetros apresentados por Santos (2011), que buscam caracterizar e qualificar ferramentas que visam suportar avaliações por inspeção no desenvolvimento de software. Tabela 1 sumariza a comparação entre a THEM e as ferramentas similares.

Tabela 1. Comparativo entre a THEM e outras ferramentas.

\begin{tabular}{|c|c|c|c|c|c|c|c|}
\hline Parâmetro & TOWABE & SUIT & APIU & MISTool & AplicSIM & HEVA & THEM \\
\hline $\begin{array}{l}\text { Foco da } \\
\text { Inspeção }\end{array}$ & $\begin{array}{c}\text { Apenas } \\
\text { Interfaces } \\
\text { de } \\
\text { Software } \\
\text { Web }\end{array}$ & \begin{tabular}{|} 
Apenas \\
Interfaces \\
de \\
Software \\
Web
\end{tabular} & $\begin{array}{c}\text { Apenas } \\
\text { Interfaces } \\
\text { de } \\
\text { Software } \\
\text { Web }\end{array}$ & $\begin{array}{c}\text { Interfaces de } \\
\text { Software } \\
\text { independente } \\
\text { da } \\
\text { plataforma }\end{array}$ & $\begin{array}{l}\text { Interfaces de } \\
\text { Software } \\
\text { independente } \\
\text { da } \\
\text { plataforma }\end{array}$ & $\begin{array}{c}\text { Apenas } \\
\text { Interfaces } \\
\text { de } \\
\text { Software } \\
\text { Web }\end{array}$ & $\begin{array}{c}\text { Interfaces de } \\
\text { Software } \\
\text { independente } \\
\text { da } \\
\text { plataforma }\end{array}$ \\
\hline $\begin{array}{l}\text { Independência } \\
\text { do Método de } \\
\text { Inspeção }\end{array}$ & Não & Não & Sim & Não & Não & Não & Não \\
\hline $\begin{array}{l}\text { Método de } \\
\text { inspeção } \\
\text { suportado }\end{array}$ & $\begin{array}{c}\text { Checklist, } \\
\text { SUE }\end{array}$ & $\begin{array}{c}\text { Checklist, } \\
\text { SUE }\end{array}$ & Todos & MIS & MIS & $\mathrm{AH}$ & $\mathrm{AH}$ \\
\hline $\begin{array}{c}\text { Voltado para } \\
\text { as } \\
\text { necessidades } \\
\text { do método }\end{array}$ & Sim & Sim & Não & Sim & Sim & Sim & Sim \\
\hline $\begin{array}{c}\text { Apoio a todo o } \\
\text { processo }\end{array}$ & Não & Sim & Sim & Não & $\begin{array}{c}\text { Não } \\
\text { informado }\end{array}$ & Não & Sim \\
\hline $\begin{array}{c}\text { Geração de } \\
\text { Relatórios }\end{array}$ & Sim & Sim & $\mathrm{Sim}$ & Não & Não & Sim & Sim \\
\hline $\begin{array}{l}\text { Executada em } \\
\text { diferentes } \\
\text { plataformas }\end{array}$ & Sim & Sim & Sim & Não & Sim & Não & Sim \\
\hline $\begin{array}{c}\text { Suporte a } \\
\text { Colaboração }\end{array}$ & $\begin{array}{c}\text { Não } \\
\text { informado }\end{array}$ & \begin{tabular}{|c|} 
Não \\
informado
\end{tabular} & $\begin{array}{c}\text { Não } \\
\text { informado }\end{array}$ & Não & Não & Não & Sim \\
\hline
\end{tabular}

A partir da Tabela 1 é possível perceber que THEM é a única ferramenta que explicitou o suporte a colaboração. Além disso, é a única ferramenta que suporta avaliações de interfaces baseadas na Avaliação Heurística $(\mathrm{AH})$, que não apresenta restrições no tipo de interface a ser avaliada e auxilia em todo o processo de execução focando nas necessidades específicas do método (e.g., geração de indicadores 
personalizados e relatórios). De forma complementar, por ser uma ferramenta Web, a THEM pode ser executada em diferentes sistemas operacionais. A seguir são apresentados os resultados do experimento que buscou avaliar a ferramenta THEM.

\section{Avaliação da THEM}

A THEM foi avaliada na perspectiva dos usuários por meio de um Teste de Usabilidade. Esta técnica consiste em um método de avaliação de usabilidade que envolve a participação de usuários e prevê as seguintes fases: preparação, execução e análise [Prates e Barbosa, 2003][Rubin e Chisnell, 2008].

A fase de preparação e subdividida nas etapas de: (1) determinação dos objetivos do teste; (2) definição das tarefas que serão executadas; (3) seleção dos participantes; (4) considerações sobre os aspectos éticos; e (5) execução do teste piloto. Essas etapas geram artefatos que são posteriormente utilizados durante o passo de execução do Teste de Usabilidade [Prates e Barbosa, 2003][Rubin e Chisnell, 2008].

A execução representa a fase em que ocorre a avaliação da usabilidade do sistema sob a perspectiva dos usuários. O avaliador conduz essa fase, seguindo os seguintes passos: (1) recebimento do usuário; (2) apresentação do sistema; (3) consentimento formal dos usuários, utilizando para isso o termo de consentimento; (4) questionamento pré-teste; (5) observação das tarefas executadas pelos usuários e (6) entrevista pós-teste. Já na terceira fase do método, os dados coletados são analisados pelos avaliadores [Prates e Barbosa, 2003][Rubin e Chisnell, 2008].

Para avaliar a usabilidade da THEM o Teste de Usabilidade foi conduzido com a participação de seis usuários que possuem formação superior na área da Computação e, pelo menos, 1 ano de experiência em avaliações de interfaces baseadas na Avaliação Heurística. Essa quantidade de usuários se justifica, uma vez que, segundo Nielsen (2000), testes de usabilidade devem ser executados por 3 a 5 usuários.

Em relação às tarefas, os usuários interagiram com os principais cenários de interação da THEM. Os cenários considerados foram: (T1) criar uma conta de usuário, (T2) efetuar login, (T3), criar um projeto de avaliação, (T4) convidar avaliadores/colaboradores para executar a avaliação, (T5) definir as heurísticas da avaliação, (T6) definir as possíveis gravidades para caracterizar os problemas identificados na avaliação, (T7) registrar dados da avaliação, (T8) gerar relatório da avaliação e (T9) exportar relatório da avaliação.

Para cada tarefa executada por um usuário, o avaliador observava e registrava como a tarefa era concluída (i.e., concluída sem erro, concluída com erro ou não concluída). Não era permitido ao longo da execução que o avaliador respondesse a perguntas referentes à interface ou a alguma funcionalidade da THEM. Este tipo de pergunta seria respondido somente no período após cada tarefa, quando também seriam discutidas as dúvidas, dificuldades e sugestões dos usuários. Cada teste foi executado em, no máximo, uma hora.

A partir dos dados obtidos, os resultados foram analisados de forma a caracterizar os indicadores de conclusão das tarefas pelos usuários e o grau de adequação da THEM aos princípios de usabilidade. Por meio dessas medidas foi possível caracterizar a usabilidade da THEM na perspectiva de seus usuários. Os resultados obtidos são discutidos a seguir. 


\subsection{Resultados da Avaliação da THEM}

Em relação à execução das tarefas, o gráfico da Figura 10 demonstra o percentual de conclusão de cada tarefa. É possível perceber que $88 \%$ das tarefas foram concluídas por todos os usuários, sendo que dessas, apenas $37 \%$ foram concluídas com erros por apenas 1 usuário, cada. Além disso, apenas a tarefa T4, deixou de ser concluída por um dos usuários.

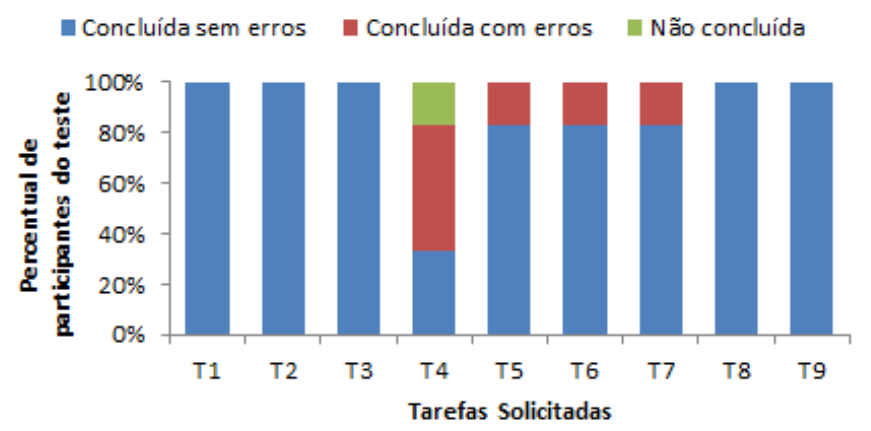

\begin{tabular}{|l|l|}
\hline \multicolumn{2}{|c|}{ Legenda } \\
\hline T1 & Criar Conta de Usuário \\
\hline T2 & Efetuar login \\
\hline T3 & Criar um projeto de avaliação \\
\hline T4 & Convidar avaliadores para a avaliação \\
\hline T5 & Definir heuristicas da avaliação \\
\hline T6 & Definir gravidades da avaliação \\
\hline T7 & Registrar dados da avaliação \\
\hline T8 & Gerar relatório da avaliação \\
\hline T9 & Exportar relatório da avaliação \\
\hline
\end{tabular}

Figura 10. Percentual de conclusão das Tarefas pelos Usuários

Finalizadas as tarefas, cada usuário foi convidado a julgar a adequação de uso da THEM sob a perspectiva dos sete princípios de usabilidade apresentados por Nielsen (1994). Para cada atributo, o participante deveria indicar se a THEM o atendia completamente, parcialmente, não atendia ou não se aplicava. A Figura 11 sumariza o resultado dessa apreciação.

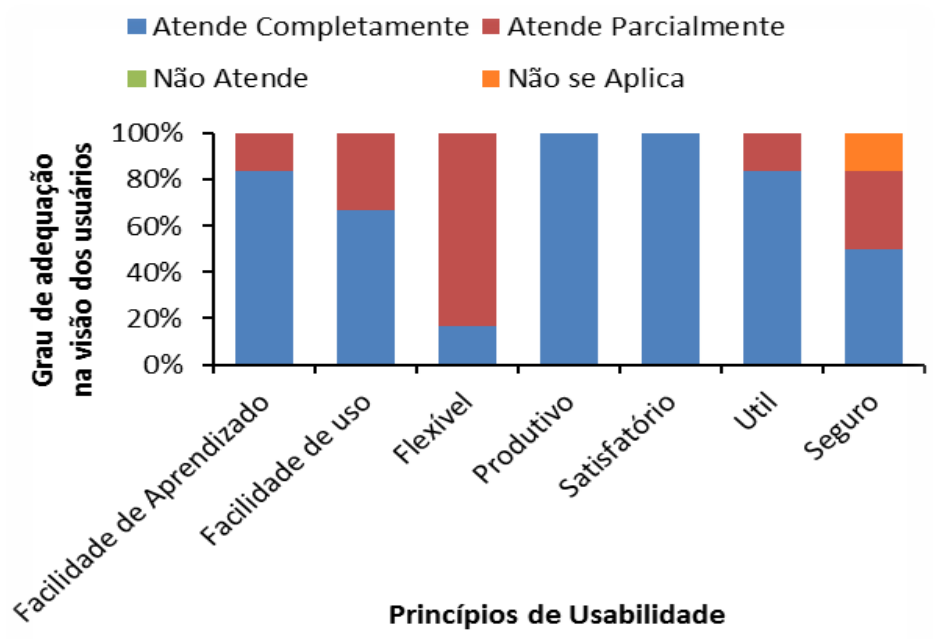

Figura 11. Grau de Adequação da THEM aos princípios de usabilidades na visão dos usuários

A partir da Figura 11 é possível perceber que na visão de, pelo menos, $80 \%$ dos usuários a THEM atende (parcialmente ou completamente) todos os princípios de usabilidade, sendo que, nenhum princípio foi indicado como violado (i.e., não atendido pela interface) e apenas 1 usuário indicou o princípio "Segurança", como não aplicável ao contexto de usabilidade da ferramenta.

O princípio flexibilidade foi o que apresentou menor adequação total na visão dos usuários. Uma possível justificativa para essa percepção refere-se ao fato da THEM 
ter sido baseada no método Avaliação Heurística (Nielsen, 1994), principal método de avaliação por inspeção guiada por heurísticas, que segue um processo sequencial para execução de suas tarefas. Contudo, será avaliada a viabilidade de melhorar a flexibilidade da THEM.

Por outro lado, pelo menos $80 \%$ dos participantes indicaram que a THEM atende completamente os princípios de facilidade de aprendizado, produtividade, satisfação e utilidade. Esses resultados reforçam a relevância da ferramenta mediante seu propósito, sobretudo na utilidade e produtividade. Isso porque, ao ser caracterizada a partir desses dois princípios é possível reforçar o argumento de que a THEM, de fato, auxilia os usuários no processo de avalição de interface por inspeção baseadas na Avaliação Heurística. Conclui-se então que, apesar das demandas por ajustes, a THEM está adequada ao uso de seus usuários.

\section{Conclusões e Trabalhos Futuros}

Esse trabalho apresentou a THEM, uma solução colaborativa que busca auxiliar no processo de avaliação de interfaces baseado na Avaliação Heurística. Em termos de resultados, além de apresentar uma análise comparativa que destaca o diferencial da THEM em relação às ferramentas existentes, o Teste de Usabilidade demonstrou que a THEM é uma ferramenta útil e satisfatória, na perspectiva de seus potenciais usuários.

Esse trabalho é relevante porque, além de suportar todas as etapas da Avaliação Heurística e contribuir com a automação de atividades previstas nesse tipo de avaliação (e.g., geração de gráficos e relatórios), a THEM permite que os avaliadores adaptem o conjunto de heurísticas e os níveis de gravidade, para cada avaliação. Esses recursos caracterizam a THEM como uma ferramenta extensível que pode ser adotada em diferentes tipos de avaliações de interfaces guiadas por heurísticas.

Além disso, a THEM motiva novos trabalhos relacionados que visam desenvolver e delinear as vantagens e desvantagens de ferramentas que suportam a execução dos métodos de avaliação de interface. De forma complementar, o levantamento das ferramentas similares também é relevante, uma vez que permite uma melhor reflexão para a escolha da ferramenta mais adequada para as diferentes abordagens de avaliação por inspeção.

Uma vez que o presente trabalho avaliou a THEM em relação a sua adequação ao uso (i.e., usabilidade) na perspectiva dos usuários, uma direção futura interessante consiste em executar experimentos que permitam caracterizar melhor a eficiência e eficácia da THEM em relação às ferramentas existentes.

\section{Referências}

Ardito, C. et al. A tool to support usability inspection. Proceedings of the working conference on Advanced visual interfaces - AVI'06, p. 278-281, 2006.

Barbosa, S. D. J. e Silva, B. S. da. Interação humano-computador. Rio de Janeiro: Elsevier, 2010.

Chaves, G. L. DE M. 2010. Desenvolvimento de software com ferramentas livres e de baixo custo: metodologia e estudo de caso. Cad. CPqD Tecnologia, v. 6, n. 1, p. 5764, 2010. 
de Souza, C. S., Leitão, C. F., Prates, R. O., and da Silva, E. J. 2006. The semiotic inspection method. In: Proc. of IHC'06, p. 148-157.

Dias, J. S. e Prates, R. 2013. O. Suporte à aplicação do método de inspeção semiótica por uma ferramenta online. In: Proc. of the 12th IHC, 2013.

Gupta, P., Toledo, R. M. e Monger M. 2012. Utilizing ASP.NET MVC in web development courses. J. Comput. Sci. Coll. 27, 3 (January 2012), 10-14.

Halling, M.; Biffl, S. e Grünbacher, P. 2002. A groupware-supported inspection process for active inspection management. Conference Proceedings of the EUROMICRO, p. 251-258, 2002.

Itakura, F. T. e Vergilio, S. R. 2002. TOWABE - Uma Ferramenta para Avaliação de Usabilidade em Aplicações para Web. Simpósio Brasileiro de Engenharia de Software, p. 462-467, 2002.

Jesus, A. M. de. e Silva, E. J. da. 2010. MISTool : um ambiente colaborativo de apoio ao Método de Inspeção Semiótica. In Proc. of IHC '10, p. 217-220, out. 2010.

Kalinowski, M.; Spínola, R. O. e Travassos, G. H. 2004. Infra-estrutura Computacional para Apoio ao Processo de Inspeção de Software. In Proc. of Simpósio Brasileiro de Qualidade de Software, p. 15, 2004.

Lanubile, F.; Mallardo, T. e Calefato, F. 2003. Tool support for geographically dispersed inspection teams. Software Process Improvement and Practice, v. 8, n. 4, p. 217-231, 2003.

Nielsen., J. 2000. Why you only need to test with 5 users. 2000. Available in: < https://goo.gl/qik9ZN>. Last access: Mar-22, 2017.

Nielsen, J. Heuristic evaluation. In: Nielsen, J. and Mack, R. L. (Eds.). Usability inspection methods. New York: John Wiley \& Sons, 1994. p. 25-62.

Pressman, R. S. Engenharia Software Pressman. 7a ed. São Paulo: Makron Books, 2011.

Prates, R. O e Barbosa, S. D. J. 2003. Avaliação de Interfaces de Usuário - Conceitos e Métodos. Jornada de Atualização em Informática (JAI), XXIII Congresso da SBC, 2003.

Oeiras, J. Y. Y., Bentolila, D. L. M. e Figueiredo, M. C. 2008. Heva: Uma Ferramenta de Suporte à Avaliação Heurística para Sistemas Web. In Proc. of VIII IHC' 08.

Rubin, J. e Chisnell, D. 2008. Handbook of usability testing: how to plan, design and conduct efective tests. John Wiley \& Sons (2008)

Santos, F. H. O. dos. 2011. Apoio ao processo de inspeção de usabilidade para aplicações de software. Dissertação (Mestrado em Informática). Manaus: Universidade Federal do Amazonas, 3 mar. 2011. 